\title{
Association between depressive symptoms and sarcopenia in older Chinese community-dwelling individuals
}

This article was published in the following Dove Press journal:

Clinical Interventions in Aging

\author{
Hui Wang ${ }^{1,2}$ \\ Shan Hai' \\ Yixin Liu' \\ Li Cao' \\ Ying Liu' \\ Ping Liu \\ Jianghua Zhou ${ }^{3}$ \\ Ying Yang ${ }^{3}$ \\ Birong Dong ${ }^{1,2}$ \\ 'Center of Gerontology and \\ Geriatrics, West China Hospital, \\ Sichuan University, Chengdu \\ 6I004I, People's Republic of China; \\ ${ }^{2}$ National Clinical Research Center \\ for Geriatrics, West China Hospital, \\ Chengdu 61004I, People's Republic \\ of China; ${ }^{3}$ Chengdu Fifth People's \\ Hospital, Chengdu 6I I I30, People's \\ Republic of China
}

Correspondence: Birong Dong National Clinical Research Center for Geriatrics, West China Hospital, No 37 Guoxue Lane, Chengdu 61004I, People's Republic of China Email birongdong@।63.com
Purpose: The aim of this study was to assess the prevalence of sarcopenia and depressive symptoms and estimate the association between them in elderly Chinese community-dwelling individuals.

Patients and methods: A total of 948 elderly Chinese community-dwelling individuals were recruited through leaflets and posters from three communities in Chengdu, and 865 participants were analyzed in this cross-sectional study. Muscle mass was measured using the bioimpedance analysis, handgrip strength was measured with a handheld dynamometer, and physical performance was assessed via usual gait speed on a $6 \mathrm{~m}$ course. Sarcopenia was defined according to the recommended diagnostic algorithm of the Asia Working Group for Sarcopenia (AWGS). Depressive symptoms were assessed using the Chinese version of 15 -item Geriatric Depression Scale (GDS-15) with a score of $\geq 5$ indicative of the presence of depressive symptoms.

Results: A total of 865 participants were included in the analysis. The participants had a mean age of $68.68 \pm 6.46$ years. Sixty-one (7.1\%) participants and $71(8.2 \%)$ participants were identified as having sarcopenia and depressive symptoms, respectively. After adjusting for age, sex, and other potential confounders, sarcopenia was found to be significantly associated with depressive symptoms (odds ratio [OR]: 2.23, 95\% CI 1.06-4.92).

Conclusion: The prevalence rates of sarcopenia and depressive symptoms were 7.1 and $8.2 \%$, respectively, in elderly Chinese community-dwelling individuals, and sarcopenia was significantly associated with depressive symptoms.

Keywords: community-dwelling, depressive symptoms, elderly, Geriatric Depression Scale, sarcopenia

\section{Introduction}

Depression, which is characterized by sadness, loss of interest or pleasure, feelings of guilt or low selfworth, disturbed sleep or appetite, feelings of tiredness, and poor concentration, is perhaps the most frequent mental disorder in later life. The total number of people living with depression worldwide was estimated by the World Health Organization to be 322 million in 2017, with an estimated prevalence in China of $\sim 4.2 \% .{ }^{1}$ Late-life depression is associated with serious consequences, including increased risk of morbidity, disability, and suicide, all of which are in turn associated with increased mortality. ${ }^{2}$ Attention has recently been given to the experience of depressive symptoms that do not fulfill the criteria for a diagnosis of major depression. A previous study reported that the prevalence of depressive symptoms among community-dwelling older adults ranged from $\sim 8$ to $16 \%,{ }^{3}$ which is much higher than the prevalence of depression. Although antidepressant medication, psychotherapy, 
or a combination of both has become the foundation for the treatment of depression in the elderly, the effectiveness of these interventions appears to be more modest in the elderly than in young adults, partly because poor health conditions and concomitant treatment with other medications may reduce the medication compliance rate of patients and influence the treatment efficacy. ${ }^{4}$ Researchers have proposed to identify the risk factors for depression in elderly individuals and, therefore, to reduce the incidence of depression through intervening on these risk factors.

Sarcopenia is a highly prevalent geriatric syndrome first described by Rosenberg in 1989 as the age-related loss of muscle mass and function. ${ }^{5}$ In 2014, the Asian Working Group for Sarcopenia (AWGS) proposed a definition for sarcopenia and recommended approaches for the measurements of muscle mass, muscle strength, and physical performance. ${ }^{6}$ According to the definition, the prevalence of sarcopenia among the general elderly population ranges from $4.1 \%$ to $11.5 \%$ in Asia. ${ }^{7}$ Sarcopenia is considered to have an association with adverse health outcomes such as frailty, falls, disability, admission to nursing homes, and mortality. Previous studies have reported a positive relationship between sarcopenia and depression in elderly Taiwanese men. ${ }^{8}$ A prospective cohort study reported that lower handgrip strength (HS) was associated with depressive symptoms in Japanese individuals. ${ }^{9}$ Additionally, Wu et al ${ }^{10}$ recently identified that depressive symptoms are inversely associated with both muscle mass and muscle strength in elderly Chinese individuals. However, some findings have been inconsistent between studies; the data from the 2010-2011 Korean National Health and Nutrition Examination Survey reported that depression was not associated with sarcopenia. ${ }^{11}$ Therefore, more population-based studies are needed to identify the real relationship between depressive syndromes and sarcopenia in elderly Chinese individuals.

In this study, we aimed to 1) evaluate the prevalence of sarcopenia according to the recommended criterion of the AWGS and the prevalence of depressive symptoms measured using the 15-item Geriatric Depression Scale (GDS-15) in elderly Chinese community-dwelling individuals and 2) investigate the association between sarcopenia and depressive symptoms.

\section{Patients and methods}

This cross-sectional study was conducted at the Yulin Community Center in Chengdu, China. The Sichuan University Ethics Committee approved this study (reference no 2014 [57]). Written informed consent was obtained from participants or their legal proxies.

\section{Participants}

Nine hundred forty-eight participants (465 men and 483 women) aged 60 years or older were recruited through leaflets and posters from three communities in Chengdu. Volunteers who self-reported the presence of disease (including hyperthyroidism, hypothyroidism, or chronic heart or renal failure), physical disabilities (eg, loss of a hand, foot, or multiple limbs), implanted electronic devices or orthopedic metal implantations, and those taking prescribed medications that could obviously affect body composition (eg, long-term systemic corticosteroids) were excluded. Individuals who could not complete the survey due to severe hearing or eye problems were also excluded from the study. Of the 948 participants recruited, one woman with missing information on skeletal muscle mass, two women with missing information from the Mini-Mental State Examination (MMSE), and 82 individuals (44 men and 38 women) with missing data from the GDS-15 were excluded. Data from a total of 865 individuals (427 men and 438 women) were analyzed.

\section{Data collection}

All interviewers participated in a half-day training workshop using investigational manuals, multimedia materials, and simulated patients. Trained interviewers collected questionnaire data through face-to-face, one-on-one personal interviews. Trained technicians performed the anthropometric and bioimpedance measurements.

\section{Assessment of sarcopenia}

According to the diagnostic algorithm of AWGS, participants with low muscle mass as well as low muscle strength or physical performance were considered to have sarcopenia. ${ }^{6}$ Muscle mass was measured by bioimpedance analysis using an Inbody 720 (BioSpace, Seoul, Korea), which was previously validated in a Chinese population. ${ }^{12}$ Low muscle mass was defined as an appendicular skeletal muscle mass index (ASMI, ASM $/$ height $^{2}$ ) of $<7.0 \mathrm{~kg} / \mathrm{m}^{2}$ in men and $<5.7 \mathrm{~kg} / \mathrm{m}^{2}$ in women. Muscle strength was assessed based on HS measured using a dynamometer (EH101; Camry, Zhongshan, China). While in a standing position, participants were asked to exert maximum effort. Three readings were taken from each side, and the maximum value from the dominant hand was used for analysis. Low muscle strength was defined as $\mathrm{HS}<26 \mathrm{~kg}$ in men and $<18 \mathrm{~kg}$ in women. The usual gait speed (GS) on a $6 \mathrm{~m}$ course was used as an objective measure of physical performance. The participants were asked to walk $6 \mathrm{~m}$ at their usual pace, and the time required to walk the distance was measured to calculate GS (m/s). Use of a cane or walker was permitted if the participant could not perform 
the gait test without it. The gait test was performed twice, and the mean value was used for analysis. Slow GS was defined as a walking speed of $\leq 0.8 \mathrm{~m} / \mathrm{s}$.

\section{Assessment of depressive symptoms}

Depressive symptoms were assessed using the Chinese short version of the GDS-15. The scale, which contains 15 items that require only a yes/no answer, is the most widely used scale for the detection of depression. It has been identified as having a high correlation with the original GDS in the Chinese population. ${ }^{13}$ In the present study, the GDS-15 was rater-administered in a standardized manner with the interviewer questioning the subjects and recording their responses to the individual items. A cutoff score of $\geq 5$ was chosen to establish the presence of depressive symptoms. ${ }^{14}$

\section{Assessment of confounders}

Demographic information included age, gender, and profession before retirement. A medical history of chronic conditions, as diagnosed by a physician, was self-reported by the participants or their caregivers. These disease conditions included thyroid diseases, heart problems, renal diseases, hypertension, stroke, cancer, diabetes, chronic obstructive pulmonary disease (COPD), hepatic diseases, and human immunodeficiency virus (HIV). Smoking status was categorized as current, former, and no smoking. Alcohol consumption status was classified as heavy (alcohol consumption $>75$ g daily), mild, and no drinking. Physical activity was assessed using the International Physical Activity Questionnaire (IPAQ) long form, which categorized participants into the following three levels: high, moderate, and low. Cognitive status was measured using the 30 -item MMSE, which is a global test with components that include orientation, attention, calculation, language, and recall. The participants were categorized as follows: severe cognitive impairment (scores ranging from 0 to 17), mild cognitive impairment (MCI) (scores ranging from 18 to 23), and normal (scores ranging from 24 to 30 ). ${ }^{15}$ In our study population, only 22 participants had scores $\leq 17$ (six men and 16 women). Therefore, we merged participants with mild and severe cognitive impairment into a single cognitive impairment group for the data analysis. Nutritional status was evaluated using the Mini Nutritional Assessment (MNA). The total score ranged from 0 to 30 , and individuals were categorized as follows: malnutrition (score $<17$ ), at risk of malnutrition (scores ranging from 17 to 23.5), and normal (scores ranging from 24 to 30). ${ }^{16}$ Fat mass was measured by bioimpedance analysis using an Inbody 720, and fat mass ratio (FMR) was calculated as the fat mass in kilograms divided by the body mass in kilograms. Venous blood samples were collected after overnight fasting and tested for each participant in the biochemistry laboratory of Sichuan University.

\section{Statistical analyses}

All statistical analyses were performed using SPSS for Windows Version 18.0 (SPSS Inc., Chicago, IL, USA). Continuous data were presented as mean \pm standard deviation (SD) if normally distributed; otherwise, they were presented as median \pm interquartile range. Categorical data are presented as absolute numbers and percentages of the total. The difference between the depressive and nondepressive groups was compared through independent $t$-tests for continuous variables with a normal distribution, and through Mann-Whitney $U$ tests for continuous data with an abnormal distribution. Pearson chi-square tests or Fisher exact tests were used for the analysis of categorical variables. Binary logistic regression models were used to estimate the odds ratio (OR) to identify associations between sarcopenia and depressive symptoms after adjusting for potential confounders. A value of $P<0.05$ was considered to be statistically significant.

\section{Results}

Overall, 865 participants (427 men and 438 women) were enrolled in the study. The participants had a mean age of $68.68 \pm 6.46$ years, and there was no significant difference in age between genders. Sixty-one (7.1\%) participants met the diagnosis of sarcopenia by the AWGS algorithm. The prevalence of sarcopenia was 6.6 and $7.5 \%$ in men and women aged 60 years or older, respectively. A total of $71(8.2 \%)$ participants were classified as having depressive symptoms, with a prevalence of 8.7 and $7.8 \%$ in men and women, respectively. Table 1 summarizes the comparisons of sociodemographic, life behavioral, clinical, and functional characteristics between participants with and without depressive symptoms. Compared to the subjects without depressive symptoms, those with depressive symptoms were less physically active and had more chronic comorbidities, especially diabetes and heart disease. The prevalence of sarcopenia in the group with depressive symptoms was significantly higher, but although the participants had lower ASMI, HS, and GS values, the differences in these values did not reach statistical significance.

We calculated the ORs using binary logistic regression models to estimate the association between depressive symptoms and sarcopenia after adjusting for age, gender, physical activity, smoking status, alcohol consumption status, cognitive condition, and body fat percentage. The results showed 
Table I Comparison of baseline characteristics between the depressive group and not depressive group

\begin{tabular}{|c|c|c|c|}
\hline Variables & No depression & Depression & $P$-value \\
\hline $\mathrm{N}$ & $794(91.8)$ & 71 (8.2) & - \\
\hline Age (years) & $68.6 \pm 6.5$ & $69.3 \pm 6.4$ & 0.398 \\
\hline Gender (female) & $404(50.9)$ & $34(47.9)$ & 0.629 \\
\hline Smoking & & & 0.171 \\
\hline Current & $127(16.0)$ & $7(9.9)$ & \\
\hline Former & 157 (I9.8) & $24(33.8)$ & \\
\hline No & $510(64.2)$ & $40(56.3)$ & \\
\hline Alcohol drinking & & & 0.021 \\
\hline Heavy & $19(2.4)$ & $5(7.0)$ & \\
\hline Mild & $198(24.9)$ & II (I5.5) & \\
\hline No & 577 (72.7) & $55(77.5)$ & \\
\hline Physical activity & & & 0.126 \\
\hline Low & $14(1.8)$ & $4(5.6)$ & \\
\hline Moderate & $254(32.0)$ & $28(39.4)$ & \\
\hline High & $525(66.2)$ & $39(54.9)$ & \\
\hline \multicolumn{4}{|l|}{ Chronic comorbidity } \\
\hline Hypertension & 340 (42.9) & $28(39.4)$ & 0.575 \\
\hline Diabetes & $136(17.1)$ & $21(29.6)$ & 0.009 \\
\hline Heart disease & $32(4.0)$ & $9(12.7)$ & 0.001 \\
\hline $\begin{array}{l}\text { Number of chronic } \\
\text { comorbidities }\end{array}$ & $0.7 \pm 0.8$ & $0.9 \pm 1.0$ & 0.040 \\
\hline Sarcopenia & $50(6.3)$ & II (I5.5) & 0.004 \\
\hline MMSE score & $26.5 \pm 3.0$ & $26.2 \pm 3.4$ & 0.379 \\
\hline GDS-I5 score & $1.2 \pm 1.2$ & $6.5 \pm 2.0$ & 0.000 \\
\hline MNA score & $27.1 \pm 2.0$ & $25.1 \pm 3.0$ & 0.000 \\
\hline BMI $\left(\mathrm{kg} / \mathrm{m}^{2}\right)$ & $24.0 \pm 3.0$ & $23.2 \pm 3.4$ & 0.032 \\
\hline SMI $\left(\mathrm{kg} / \mathrm{m}^{2}\right)$ & $6.6 \pm 1.0$ & $6.5 \pm 1.1$ & 0.482 \\
\hline BFP (\%) & $31.6 \pm 7.4$ & $29.9 \pm 7.3$ & 0.049 \\
\hline HS (kg) & $30.1 \pm 8.9$ & $29.2 \pm 9.4$ & 0.404 \\
\hline $\mathrm{GS}(\mathrm{m} / \mathrm{s})$ & $1.0 \pm 0.2$ & $1.0 \pm 0.2$ & 0.051 \\
\hline
\end{tabular}

Notes: Using independent-samples t-test for continuous variables and Pearson chisquare or Fisher's exact test for categorical variables. During testing, $P<0.05$ was considered statistically significant. Data are presented as mean \pm standard deviation or $\mathrm{n}(\%)$.

Abbreviations: BFP, body fat percentage; BMI, body mass index; GDS-15, I5-item Geriatric Depression Scale; GS, gait speed; HS, handgrip strength; MMSE, MiniMental State Examination; MNA, Mini Nutritional Assessment; SMI, appendicular skeletal muscle mass index.

that sarcopenia was significantly associated with depressive symptoms in both the unadjusted model (OR: 2.72, 95\% CI, 1.35-5.51) and the fully adjusted model (OR: $2.23,95 \% \mathrm{CI}$, 1.06-4.92) (Table 2).

\section{Discussion}

Our findings revealed a significant association between sarcopenia and the presence of depressive symptoms in the elderly Chinese community-dwelling population, independent of age, gender, cognitive function, and other potential confounders. The results were similar to the findings of previous cross-sectional studies on the association between depressive symptoms and sarcopenia. Hsu et al, ${ }^{8}$ whose study focused on elderly Asian individuals, reported that sarcopenia was significantly associated with depressive symptoms in Taiwanese individuals. Moreover, Kim et $\mathrm{al}^{17}$ suggested that sarcopenia was closely associated with an increased risk of suicidal ideation in elderly Korean men. A meta-analysis comprising 15 observational studies also indicated that patients with sarcopenia were likely to present with depression. ${ }^{18}$ However, the average age of the population ranged from 43.3 to 86.7 years and the diagnostic algorithms for sarcopenia and depression were diverse between the 15 studies. Moreover, in the 2010-2011 Korean National Health and Nutrition Examination Survey, Byeon et $\mathrm{al}^{11}$ concluded that sarcopenia was not associated with depression. In this study, the author defined sarcopenia only as a decrease in muscle mass rather than the combination of changes in muscle mass and muscle function, which is significantly different from the definitions used in our study. Numerous studies also investigated the relationship between muscle mass, muscle strength, and depressive symptoms; however, the results have been controversial. A Korean study reported that low muscle mass was related to depression in elderly individuals. ${ }^{19}$ Reduction in grip strength was also reported to be associated with a higher risk of depressive symptoms. ${ }^{9,20}$ In a recent cross-sectional survey, Wu et al ${ }^{10}$ identified that both muscle mass and muscle strength were inversely associated with depressive symptoms in elderly Chinese individuals. Additionally, sarcopenic obesity was demonstrated to be positively associated with depressive symptoms ${ }^{21}$ and lower indicators of psychological health,

Table 2 Association between depressive symptoms and sarcopenia according to logistic regression models adjusted for confounders

\begin{tabular}{|c|c|c|c|c|}
\hline Variables & Unadjusted & Model I & Model 2 & Model 3 \\
\hline Sarcopenia & $2.73(|.35-5.5|)$ & $2.69(1.29-5.63)$ & $2.74(1.28-5.84)$ & $2.45(1.12-5.34)$ \\
\hline Age (years) & & $1.00(0.97-1.04)$ & $0.99(0.95-1.03)$ & $0.99(0.95-1.03)$ \\
\hline Gender (female) & & $0.88(0.54-1.43)$ & $0.7 \mid(0.4 I-I .22)$ & $1.03(0.54-1.97)$ \\
\hline Smoker & & & $0.42(0.18-0.99)$ & $0.39(0.16-0.93)$ \\
\hline Alcohol drinker & & & $0.74(0.39-1.37)$ & $0.76(0.4 I-I .42)$ \\
\hline Physical activity & & & $0.60(0.38-0.94)$ & $0.57(0.36-0.90)$ \\
\hline Cognitive impairment & & & & $\mathrm{I} .40(0.7 \mathrm{I}-2.77)$ \\
\hline Body fat percentage & & & & $0.95(0.92-0.99)$ \\
\hline
\end{tabular}

Notes: Data are presented as odds ratio ( $95 \%$ confidential intervals). Model I: adjusted for age and gender. Model 2: adjusted for age, gender, smoking status, alcohol drinking status, and physical activity. Model 3: adjusted for age, gender, smoking status, alcohol drinking status, physical activity, cognitive impairment, and body fat percentage. 
including perceived stress and suicidal ideation. ${ }^{22}$ The consistency in the results of these studies indicates that there are likely several similar underlying mechanisms between sarcopenia and depressive symptoms.

First, low-grade inflammation is considered to be associated with both sarcopenia and depression. The role of cytokines in depression was first proposed by $\mathrm{Smith}^{23}$ in the form of the "macrophage theory of depression" and further studied by Maes et $\mathrm{al}^{24}$ in the early 1990s. Inflammation has been identified as an important biological event that might increase the risk of depression. A meta-analysis found some proinflammatory cytokines, including interleukin-6 (IL-6) and tumor necrosis factor-alpha (TNF- $\alpha$ ), to be associated with depressive symptoms. ${ }^{25}$ Furthermore, in a prospective double-blind study, researchers identified that the combination of reboxetine (a selective noradrenergic reuptake inhibitors) and celecoxib (a cyclooxygenase-2 [COX-2] inhibitor) resulted in significantly greater improvement compared with a reboxetine-only group in patients with major depression. ${ }^{26}$ Inflammation has also been identified to be associated with sarcopenia, particularly sarcopenic obesity. Schaap et $\mathrm{al}^{27}$ reported that TNF- $\alpha$ showed consistent associations with the decline in muscle mass and strength at a 5-year follow-up. Previous studies have also proposed that a proinflammatory state might be one of the key factors creating a vicious cycle of sarcopenic obesity via a decrease in muscle mass and muscle strength in obese individuals. ${ }^{28}$ Therefore, inflammation is considered as one kind of common etiopathogenesis for sarcopenia and depression. Tryptophan is another possible underlying mechanism. Depressive symptoms are associated with reduced plasma levels of tryptophan. ${ }^{29}$ The decrease in plasma levels of tryptophan could be due to activation of the major enzymes that metabolize tryptophan, namely tryptophan 2,3 dioxygenase (TDo) and indoleamine 2,3 dioxygenase (IDo). Both enzymes degrade tryptophan via the kynurenine pathway, and IDo can be activated by a number of cytokines, including interferon- $\gamma$ (IFN- $\gamma$ ) and TNF- $\alpha$. Recently, tryptophan also was considered to be associated with sarcopenia. Low concentrations of tryptophan were reported to be significantly associated with sarcopenia in a univariate analysis, but not in a multivariable analysis. ${ }^{30}$ Additionally, N-terminal tryptophan residues were highlighted as being important to the inhibition of myostatin, which can negatively regulate skeletal muscle growth. ${ }^{31}$ In the present study, we did not measure the plasma levels of cytokines or tryptophan; therefore, elucidation of the mechanism by which sarcopenia affects depressive symptoms requires further study.
In the present study, a considerable number of potential confounders, including age, gender, smoking status, alcohol consumption status, physical activity, cognitive function, and body fat percentage, were analyzed in a logistic regression. The observed association between sarcopenia and depressive symptoms did not vanish, which suggests that these confounders only partly altered the interpretation of the relationship. Previous studies have concluded that depression and dementia are common comorbidities because the two diseases result from the same neuropathological changes. ${ }^{32,33}$ Geda et $\mathrm{al}^{34}$ followed a prospective cohort free of both depression and cognitive impairment and found that individuals who developed depression were more likely to subsequently develop MCI or dementia. However, controversy remains as to whether depression is a risk factor for dementia or merely a prodromal feature of dementia. Some researchers have proposed that dementia is a risk factor for depression and that the depressive symptoms are a psychological reaction to the cognitive and behavioral changes accompanying dementia. ${ }^{35}$ However, we failed to identify any significant association in the present study. The difference may be due in part to the limitation of our population. The cognitive status of our participants was relatively normal, with only 22 participants having MMSE scores $\leq 17$. Our population might not have been adequate to allow us to properly estimate the relationship between depression and cognitive impairment. In future studies, participants with various cognitive statuses should be recruited and a prospective cohort study should be performed to elucidate the relationship between depression and cognitive impairment.

Our study had several limitations. First, we recruited participants through leaflets and posters instead of by random selection. This recruitment method resulted in the exclusion of elderly individuals with severe frailty or depression and inevitably led to a selection bias. Meanwhile, we used a variety of questionnaires in present study; therefore, the self-report bias and recall bias were unavoidable. We may have underestimated the prevalence of sarcopenia and depressive symptoms, thereby affecting the results of the analysis. Second, we excluded 83 participants because of missing data. Although the included and excluded individuals were similar in age, gender, and lifestyle, the exclusion of participants may have also resulted in a selection bias. Third, the cross-sectional nature of this study did not allow us to identify the causal relationships as in a longitudinal or semilongitudinal study. We are only able to hypothesize the causal relationship between sarcopenia and depression. Fourth, the depressive symptoms were measured using the rater-administered GDS-15; however, the GDS-15 is only 
a depression screening tool and not a diagnostic instrument. Therefore, the association between sarcopenia and depression remains to be completely established and further investigation using standard diagnostic criteria is required. Fifth, many confounders, such as disability, frailty, social support, and educational level, might also affect the association between sarcopenia and depression; however, we could not contain all the confounders in the present study. In future studies, we would analyze more confounders according to the characters of participants.

\section{Conclusion}

This study demonstrated that the prevalence of sarcopenia or depressive symptoms was high in elderly Chinese community-dwelling individuals and that sarcopenia is significantly associated with depressive symptoms. Considering the health consequences of depressive symptoms is increasingly being recognized, and the possibility of reducing the incidence of depression through intervening sarcopenia, prospective studies with large sample sizes are needed to elucidate the causal relationship between both clinical conditions.

\section{Acknowledgments}

The authors would like to thank the staff of the Department of Geriatrics Medicine of the West China Hospital, the postgraduates of Professor Birong Dong, and the staff of Yulin Community Hospital. We also thank Professor Guanjian Liu for statistical analysis. This research was supported by Abbott Nutrition (NCT02089906), Sichuan Provincial Science and Technology Department (2018FZ0064), and the Project of Benefit the People of The Technology Bureau in Chengdu (2015-HM01-00417-SF).

\section{Author contributions}

All authors contributed toward data analysis, drafting and revising the paper and agree to be accountable for all aspects of the work.

\section{Disclosure}

The authors report no conflicts of interest in this work.

\section{References}

1. WHO. Depression and Other Common Mental Disorders: Global Health Estimates. Geneva: World Health Organization; 2017.

2. Alexopoulos GS. Depression in the elderly. Lancet. 2005;365(9475): 1961-1970.

3. Blazer DG. Depression in late life: review and commentary. J Gerontol A Biol Sci Med Sci. 2003;58(3):M249-M265.
4. Mccusker J, Cole M, Keller E, Bellavance F, Berard A. Effectiveness of treatments of depression in older ambulatory patients. Arch Intern Med. 1998;158(7):705-712.

5. Rosenberg IH. Sarcopenia: origins and clinical relevance. $J$ Nutr. 1997;127(5 suppl):990S-991S.

6. Cruz-Jentoft AJ, Landi F, Schneider SM, et al. Prevalence of and interventions for sarcopenia in ageing adults: a systematic review. Report of the International Sarcopenia Initiative (EWGSOP and IWGS). Age Ageing. 2014;43(6):748-759.

7. Chen L-K, Lee W-J, Peng L-N, et al. Recent advances in sarcopenia research in Asia: 2016 update from the Asian Working Group for Sarcopenia. J Am Med Dir Assoc. 2016;17(8):767.

8. Hsu YH, Liang CK, Chou MY, et al. Association of cognitive impairment, depressive symptoms and sarcopenia among healthy older men in the veterans retirement community in southern Taiwan: a cross-sectional study. Geriatr Gerontol Int. 2014;14(suppl 1):102-108.

9. Fukumori N, Yamamoto Y, Takegami M, et al. Association between hand-grip strength and depressive symptoms: locomotive syndrome and health outcomes in Aizu Cohort Study (LOHAS). Age Ageing. 2015;44(4):592-598.

10. Wu H, Yu B, Meng G, et al. Both muscle mass and muscle strength are inversely associated with depressive symptoms in an elderly Chinese population. Int J Geriatr Psychiatry. 2017;32(7):769-778.

11. Byeon CH, Kang KY, Kang SH, Kim HK, Bae EJ. Sarcopenia is not associated with depression in Korean adults: results from the 2010-2011 Korean National Health and Nutrition Examination Survey. Korean $J$ Fam Med. 2016;37(1):37-43.

12. Wang H, Hai S, Cao L, Zhou J, Liu P, Dong BR. Estimation of prevalence of sarcopenia by using a new bioelectrical impedance analysis in Chinese community-dwelling elderly people. BMC Geriatr. 2016;16(1):216.

13. Lee HCB, Chiu HFK, Kwok WY, Leung CM, Kwong PK, Chung DWS. Chinese elderly and the GDS short form: a preliminary study. Clin Gerontol. 1993;14:37-42.

14. Lim PP, Ng LL, Chiam PC, Ong PS, Ngui FTS, Sahadevan S. Validation and comparison of three brief depression scales in an elderly Chinese population. Int J Geriatr Psychiatry. 2000;15(9):824-830.

15. Tombaugh TN, Mcintyre NJ. The mini-mental state examination: a comprehensive review. J Am Geriatr Soc. 1992;40(9):922-935.

16. Guigoz Y, Vellas B, Garry PJ. Assessing the nutritional status of the elderly: the Mini Nutritional Assessment as part of the geriatric evaluation. Nutr Rev. 1996;54(1 pt 2):S59-S65.

17. Kim JH, Kim DH, Park YS, Composition B. Body composition, sarcopenia, and suicidal ideation in elderly Koreans: Hallym Aging Study. J Korean Med Sci. 2016;31(4):604-610.

18. Chang KV, Hsu TH, Wu WT, Huang KC, Han DS. Is sarcopenia associated with depression? A systematic review and meta-analysis of observational studies. Age Ageing. 2017;46(5):738-746.

19. Kim NH, Kim HS, Eun CR, et al. Depression is associated with sarcopenia, not central obesity, in elderly Korean men. J Am Geriatr Soc. 2011;59(11):2062-2068.

20. Hamer M, Batty GD, Kivimaki M. Sarcopenic obesity and risk of new onset depressive symptoms in older adults: English Longitudinal Study of Ageing. Int J Obes. 2015;39(12):1717-1720.

21. Ishii $\mathrm{S}$, Chang $\mathrm{C}$, Tanaka $\mathrm{T}$, et al. The association between sarcopenic obesity and depressive symptoms in older Japanese adults. PLoS One. 2016;11(9):e0162898.

22. Cho Y, Shin SY, Shin MJ. Sarcopenic obesity is associated with lower indicators of psychological health and quality of life in Koreans. Nutr Res. 2015;35(5):384-392.

23. Smith RS. The macrophage theory of depression. Med Hypotheses. 1991;35(4):298-306.

24. Maes M, Bosmans E, Suy E, Vandervorst C, de Jonckheere C, Raus J. Immune disturbances during major depression: upregulated expression of interleukin-2 receptors. Neuropsychobiology. 1990;24(3): 115-120. 
25. Dowlati Y, Herrmann N, Swardfager W, et al. A meta-analysis of cytokines in major depression. Biol Psychiatry. 2010;67(5):446-457.

26. Müller N, Schwarz MJ, Dehning S, et al. The cyclooxygenase-2 inhibitor celecoxib has therapeutic effects in major depression: results of a double-blind, randomized, placebo controlled, add-on pilot study to reboxetine. Mol Psychiatry. 2006;11(7):680-684.

27. Schaap LA, Pluijm SM, Deeg DJ, et al. Higher inflammatory marker levels in older persons: associations with 5-year change in muscle mass and muscle strength. J Gerontol A Biol Sci Med Sci. 2009;64(11): 1183-1189.

28. Stenholm S, Harris TB, Rantanen T, et al. Cause and consequences. Cur Opin Clin Nutr Metab Care. 2008;11(6):693-700.

29. Strasser B, Gostner JM, Fuchs D, Mood FD. Mood, food, and cognition: role of tryptophan and serotonin. Curr Opin Clin Nutr Metab Care. 2016;19(1):55-61.

30. Toyoshima K, Nakamura M, Adachi $Y$, et al. Increased plasma proline concentrations are associated with sarcopenia in the elderly. PLoS One. 2017;12(9):e0185206.
31. Takayama K, Nakamura A, Rentier C, et al. Effect of N-terminal acylation on the activity of myostatin inhibitory peptides. ChemMedChem. 2016;11(8):845-849.

32. Jorm AF. History of depression as a risk factor for dementia: an updated review. Aust N Z J Psychiatry. 2001;35(6):776-781.

33. Ownby RL, Crocco E, Acevedo A, John V, Loewenstein D. Depression and risk for Alzheimer disease: systematic review, meta-analysis, and metaregression analysis. Arch Gen Psychiatry. 2006;63(5):530-538.

34. Geda YE, Knopman DS, Mrazek DA, et al. Depression, apolipoprotein E genotype, and the incidence of mild cognitive impairment: a prospective cohort study. Arch Neurol. 2006;63(3):435-440.

35. Fiske A, Wetherell JL, Gatz M. Depression in older adults. Annu Rev Clin Psychol. 2009;5:363-389.
Clinical Interventions in Aging

\section{Publish your work in this journal}

Clinical Interventions in Aging is an international, peer-reviewed journal focusing on evidence-based reports on the value or lack thereof of treatments intended to prevent or delay the onset of maladaptive correlates of aging in human beings. This journal is indexed on PubMed Central, MedLine,

\section{Dovepress}

CAS, Scopus and the Elsevier Bibliographic databases. The manuscript management system is completely online and includes a very quick and fair peer-review system, which is all easy to use. Visit http://www.dovepress. $\mathrm{com} /$ testimonials.php to read real quotes from published authors. 